\title{
Waveform inversion using a logarithmic wavefield
}

\author{
Changsoo Shin ${ }^{1}$ and Dong-Joo Min $^{2}$
}

\begin{abstract}
Although waveform inversion has been studied extensively since its beginning 20 years ago, applications to seismic field data have been limited, and most of those applications have been for global-seismology- or engineering-seismology-scale problems, not for exploration-scale data. As an alternative to classical waveform inversion, we propose the use of a new, objective function constructed by taking the logarithm of wavefields, allowing consideration of three types of objective function, namely, amplitude only, phase only, or both. In our waveform inversion, we estimate the source signature as well as the velocity structure by including functions of amplitudes and phases of the source signature in the objective function. We compute the steepest-descent directions by using a matrix formalism derived from a frequency-domain, finite-element/finite-difference modeling technique. Our numerical algorithms are similar to those of reverse-time migration and waveform inversion based on the adjoint state of the wave equation. In or-
\end{abstract}

der to demonstrate the practical applicability of our algorithm, we use a synthetic data set from the Marmousi model and seismic data collected from the Korean continental shelf. For noisefree synthetic data, the velocity structure produced by our inversion algorithm is closer to the true velocity structure than that obtained with conventional waveform inversion. When random noise is added, the inverted velocity model is also close to the true Marmousi model, but when frequencies below $5 \mathrm{~Hz}$ are removed from the data, the velocity structure is not as good as those for the noise-free and noisy data. For field data, we compare the time-domain synthetic seismograms generated for the velocity model inverted by our algorithm with real seismograms and find that the results show that our inversion algorithm reveals short-period features of the subsurface. Although we use wrapped phases in our examples, we still obtain reasonable results. We expect that if we were to use correctly unwrapped phases in the inversion algorithm, we would obtain better results.

\section{INTRODUCTION}

Waveform-inversion and traveltime tomography provide detailed subsurface-velocity information. Because Lailly (1983) and Tarantola (1984) suggested that the back-propagation algorithm of reverse-time migration can be used in seismic inversion, their ideas have commonly been used in time-domain traveltime tomography inversion and waveform inversion (Bamberger et al., 1982; Kolb et al., 1986; Gauthier et al., 1986; Tarantola, 1986, 1987; Tarantola et al., 1988; Mora, 1987, 1989; Sun and McMechan, 1992; Cao et al., 1990; Pica et al., 1990; Xu et al., 1995; Zhou et al., 1995). After Pratt et al. (1998) applied the same idea to frequency-domain waveform inversion and showed that the back-propagation algorithm could be used efficiently for waveform inversion of largescale geologic models, the technique began to be used frequently (Pratt, 1999; Pratt and Shipp, 1999; Hicks and Pratt, 2001). Ac- cording to Lailly (1983), prestack reverse-time migration can be regarded as the first iteration result of waveform inversion, so waveform inversion and prestack reverse-time migration share the numerical algorithm that originates from the symmetry of the wave equation Green's function.

Although a variety of waveform-inversion techniques has been developed, we need more accurate or efficient algorithms. We modify a waveform-inversion algorithm by using the logarithmic wavefield as an objective function. By taking the logarithm of the wavefield, we separate amplitude and phase, allowing us to construct three kinds of objective functions using amplitude only, phase only, or both. We can invert amplitude and phase, either separately or simultaneously. Our waveform-inversion algorithm also includes inversion of the source signature. In real seismic data, because the source signature is usually unknown, we need to estimate the source signature simultaneously (Pratt, 1999) with veloc-

Manuscript received by the Editor August 11, 2003; revised manuscript received September 6, 2005; published online May $24,2006$.

${ }^{1}$ Seoul National University, School of Civil, Urban and Geosystem Engineering, San 56-1, Shillim-dong, Kwanak-ku, Seoul, 151-744, Korea. E-mail: css@model.snu.ac.kr.

${ }^{2}$ Korea Ocean Research and Development Institute, Ansan, P. O. Box 29, Seoul, 425-600, Korea. E-mail: djmin@kordi.re.kr. (C) 2006 Society of Exploration Geophysicists. All rights reserved. 
ity inversion. Explicitly expressing the amplitude and phase of the source signature in our objective function allows us to recover the amplitude and phase of the source signature separately.

We begin by constructing an objective function that is separable into three types. We implicitly calculate gradients of the three kinds of objective functions by using the adjoint state of the wave equation as Tarantola (1984) and Pratt et al. (1998) did in conventional seismic waveform inversion. Then we combine the estimation of the source wavelet with the implicit calculation of the gradient vector of the $l_{2}$ norm of seismic-data residuals. Finally, we demonstrate our approach using the Marmousi synthetic data and real seismic data collected on the Korean continental shelf.

\section{WAVEFORM-INVERSION ALGORITHM}

Our algorithm uses the steepest-descent method to iteratively update model parameters until we satisfy a convergence criterion for an objective function to be either minimized or maximized. Conventionally, to apply the steepest-descent method to an optimization problem, we need to directly calculate the derivative of the objective function with respect to the model parameters. In this study, we construct an objective function that is separable into three types (using amplitude only, phase only, and both) and use an implicit calculation of the steepest-descent direction (i.e., without directly computing Fréchet derivatives). This is accomplished by

Table 1. Symbols.

\begin{tabular}{|c|c|c|}
\hline Symbol & Type & Description \\
\hline$u(t)$ & real & Time-domain pressure or displacement \\
\hline$\tilde{u}(\omega)$ & complex & Fourier-transformed, forward-modeled data \\
\hline$\tilde{d}(\omega)$ & complex & Fourier-transformed field data \\
\hline $\mathbf{S}(\omega)$ & complex & $\begin{array}{l}\text { Complex impedance matrix and forward-modeling } \\
\text { operator }\end{array}$ \\
\hline $\mathbf{f}(z)$ & real & Source vector in depth \\
\hline$g(\omega)$ & complex & Fourier-transformed source function \\
\hline$g_{s}(\omega), \theta_{s}(\omega)$ & real & Amplitude and phase of source wavelet \\
\hline$A^{m}(\omega), \theta^{m}(\omega)$ & real & Amplitude and phase of forward-modeled data \\
\hline$A^{f}(\omega), \theta^{f}(\omega)$ & real & Amplitude and phase of field data \\
\hline$E$ & real & Objective function \\
\hline p & real & Velocity-parameter vector \\
\hline$\delta \mathbf{p}$ & real & Velocity-parameter change vector \\
\hline $\mathbf{p}_{\mathrm{s}}$ & real & $\begin{array}{l}\text { Change vector in amplitude and phase of source } \\
\text { signature }\end{array}$ \\
\hline$\phi, \nabla E$ & real & $\begin{array}{l}\text { Gradient vector of the objective function with } \\
\text { respect to velocity parameters }\end{array}$ \\
\hline $\mathbf{v}(\omega)$ & complex & Virtual-source vector \\
\hline $\mathbf{V}(\omega)$ & complex & Virtual-source matrix \\
\hline $\mathbf{r}(\omega)$ & complex & Residual vector \\
\hline$\nabla_{s} E$ & real & $\begin{array}{l}\text { Gradient vector with respect to amplitude and phase } \\
\text { of source wavelet }\end{array}$ \\
\hline $\mathbf{H}_{s}$ & real & $\begin{array}{l}\text { Hessian matrix with respect to amplitude and phase } \\
\text { of source wavelet }\end{array}$ \\
\hline $\mathbf{J}$ & complex & Jacobian matrix \\
\hline$\lambda$ & real & Damping factor \\
\hline I & real & Identity matrix \\
\hline
\end{tabular}

using the back-propagation technique of reverse-time migration based on the adjoint state of the wave equation (e.g., Pratt et al., 1998).

For simplicity, we begin with a 1D model, because it is straightforward to extend our algorithm to $2 \mathrm{D}$ or $3 \mathrm{D}$ problems. Mathematical symbols are presented in Table 1 . The 1D constant-density acoustic-wave equation can be written as

$$
\frac{\partial^{2} u(z, t)}{\partial z^{2}}=\frac{1}{v(z)^{2}} \frac{\partial^{2} u(z, t)}{\partial t^{2}}+f(z) g(t)
$$

where $v(z)$ is the subsurface velocity function, $u(z, t)$ is the acoustic-pressure field, and $f(z)$ and $g(t)$ are the source functions in position and time, respectively. Equation 1 can be solved by finite-difference, time-marching techniques (Alford et al., 1974) or frequency-domain methods (Marfurt, 1984; Marfurt and Shin, 1989; Jo et al., 1996; Shin and Sohn, 1998). In the frequency domain, we take the Fourier transform of equation 1 and then solve the discretized matrix equation using frequency-domain, finitedifference, or finite-element modeling methods. Doing this, we obtain the matrix equation (Marfurt, 1984)

$$
\mathbf{S}(\omega) \widetilde{\mathbf{u}}(\omega)=\mathbf{f}(z) g(\omega),
$$

where $\omega$ is the angular frequency, $\mathbf{S}(\omega)$ is the complex impedance matrix, $\widetilde{\mathbf{u}}(\omega)$ is the Fourier-transformed wavefield, $\mathbf{f}(z)$ is the vector denoting source position in depth, and $g(\omega)$ is the source function in the frequency domain. For simplicity, we write the complex source function $g(\omega)$ of equation 2 as $g_{s}(\omega) \exp \left[i \theta_{s}(\omega)\right]$, yielding

$$
\mathbf{S}(\omega) \widetilde{\mathbf{u}}(\omega)=\mathbf{f}(z) g_{s}(\omega) \exp \left[i \theta_{s}(\omega)\right],
$$

where $g_{s}(\omega)$ and $\theta_{s}(\omega)$ are the amplitude and phase spectra of the source wavelet, respectively.

The 1D earth model is subdivided into a finite-difference grid in the $z$-direction $[z=(j-1) \Delta z ; j=1,2, \ldots, N]$ and each nodal point is parametrized with velocity $p_{k}$ at the $k$ th depth point. The $1 \mathrm{D}$ wavefield at each frequency can be expressed from the linearity of the wave equation with respect to the source as

$$
\begin{aligned}
\tilde{u}_{j}(\omega)= & g_{s}(\omega) A_{j}^{m}(\omega) \exp \left[i \theta_{j}^{m}(\omega)\right. \\
& \left.+i \theta_{s}(\omega)\right],
\end{aligned}
$$

where $\widetilde{u}_{j}(\omega)$ is the wavefield at the $j$ th depth point, $A_{j}^{m}(\omega)$ and $\theta_{j}^{m}(\omega)$ are the amplitude and phase of the Green's function at the $j$ th depth point, and $\theta_{s}(\omega)$ is the 
phase spectrum of the source signature. The superscript $m$ is used to discriminate modeled data from field data (which is represented using the superscript $f$ ). The modeled wavefield at the surface is

$$
\tilde{u}_{1}(\omega)=g_{s}(\omega) A_{1}^{m}(\omega) \exp \left[i \theta_{1}^{m}(\omega)+i \theta_{s}(\omega)\right]
$$

Similarly, the measured field seismogram at the surface is

$$
\tilde{d}_{1}(\omega)=A_{1}^{f}(\omega) \exp \left[i \theta_{1}^{f}(\omega)\right]
$$

where $\tilde{d}_{1}(\omega)$ is the wavefield measured at the surface and $A_{1}^{f}(\omega)$ and $\theta_{1}^{f}(\omega)$ are the amplitude and the phase of the wavefield measured at the surface, respectively. However, when we extract the phase information from the measured or modeled wavefields, we actually obtain the wrapped phases (unless we apply an unwrapping algorithm). As a result, we rewrite equations 5 and 6 as

$\tilde{u}_{1}(\omega)=g_{s}(\omega) A_{1}^{m}(\omega) \exp \left[i\left(\theta_{1}^{m}(\omega)+2 \pi n_{m}+\theta_{s}(\omega)+2 \pi n_{s}\right)\right]$

and

$$
\tilde{d}_{1}(\omega)=A_{1}^{f}(\omega) \exp \left[i\left(\theta_{1}^{f}(\omega)+2 \pi n_{f}\right)\right]
$$

respectively, where $n_{m}, n_{s}$, and $n_{f}$ are integer values that must be extracted.

In our new waveform-inversion algorithm, we construct an objective function based on the $l_{2}$ norm of residuals between logarithmic-modeled wavefields and field wavefields. This residual at the surface can be expressed as

$$
\begin{aligned}
\ln \frac{\tilde{u}_{1}(\omega)}{\tilde{d}_{1}(\omega)}= & \ln \frac{g_{s}(\omega) A_{1}^{m}(\omega)}{A_{1}^{f}(\omega)}+i\left[\theta_{1}^{m}(\omega)+\theta_{s}(\omega)-\theta_{1}^{f}(\omega)\right. \\
& \left.+2 \pi\left(n_{m}+n_{s}-n_{f}\right)\right] .
\end{aligned}
$$

As shown in equation 9, in order to obtain meaningful phase information, we need to unwrap the phase, but it is generally difficult to accomplish this unambiguously. We assume that $n_{m}+n_{s}=n_{f}$. The assumption that the phase of the field data is unwrapped to the same degree as the phase of the model data is equivalent to assuming that the model is close to the true model. The objective function can then be defined for a single frequency as

$$
E=\frac{1}{2}\left\{\left[\ln \frac{g_{s}(\omega) A_{1}^{m}(\omega)}{A_{1}^{f}(\omega)}\right]^{2}+\left[\theta_{1}^{m}(\omega)+\theta_{s}(\omega)-\theta_{1}^{f}(\omega)\right]^{2}\right\},
$$

where the factor $1 / 2$ is used to simplify the resulting equation. From equation 10, we note that the objective function is represented as the $l_{2}$ norm of the combined phase- and logarithmicamplitude errors. The gradient of this objective function with respect to the $k$ th velocity parameter $p_{k}$ is

$$
\begin{aligned}
\phi_{k}= & \nabla_{p_{k}} E=\frac{\partial E}{\partial p_{k}}=\ln \left(\frac{g_{s}(\omega) A_{1}^{m}(\omega)}{A_{1}^{f}(\omega)}\right)\left(\frac{1}{A_{1}^{m}(\omega)}\right) \frac{\partial A_{1}^{m}(\omega)}{\partial p_{k}} \\
& +\left[\theta_{1}^{m}(\omega)+\theta_{s}(\omega)-\theta_{1}^{f}(\omega)\right] \frac{\partial \theta_{1}^{m}(\omega)}{\partial p_{k}}
\end{aligned}
$$

A straightforward technique for calculating the gradient is to compute and use the partial-derivative wavefield. The partial-derivative wavefield with respect to $p_{k}$ is expressed from equation 5 as

$$
\begin{aligned}
\frac{\partial \tilde{u}_{1}(\omega)}{\partial p_{k}}= & g_{s}(\omega) \frac{\partial A_{1}^{m}(\omega)}{\partial p_{k}} \exp \left[i \theta_{1}^{m}(\omega)+i \theta_{s}(\omega)\right] \\
& +i g_{s}(\omega) A_{1}^{m}(\omega) \frac{\partial \theta_{1}^{m}(\omega)}{\partial p_{k}} \exp \left[i \theta_{1}^{m}(\omega)+i \theta_{s}(\omega)\right],
\end{aligned}
$$

and dividing equation 12 by equation 5 gives

$$
\frac{1}{\tilde{u}_{1}(\omega)} \frac{\partial \tilde{u}_{1}(\omega)}{\partial p_{k}}=\frac{1}{A_{1}^{m}(\omega)} \frac{\partial A_{1}^{m}(\omega)}{\partial p_{k}}+i \frac{\partial \theta_{1}^{m}(\omega)}{\partial p_{k}} .
$$

We recognize that the terms on the right-hand side of equation 11 can be obtained by taking the conjugate of equation 9, multiplying it by equation 13, and taking the real part of the result. Thus,

$$
\phi_{k}=\operatorname{Re}\left\{\left[\ln \frac{\tilde{u}_{1}(\omega)}{\tilde{d}_{1}(\omega)}\right]^{*} \frac{1}{\tilde{u}_{1}(\omega)} \frac{\partial \tilde{u}_{1}(\omega)}{\partial p_{k}}\right\}
$$

where ${ }^{*}$ indicates the complex conjugate. Equation 14 for an entire frequency band can be rewritten in matrix form as

$$
\begin{aligned}
\phi_{k}= & \operatorname{Re} \int_{-\infty}^{\infty}\left[\frac{\partial \tilde{u}_{1}(\omega)}{\partial p_{k}} \frac{\partial \tilde{u}_{2}(\omega)}{\partial p_{k}} \cdots \frac{\partial \tilde{u}_{N}(\omega)}{\partial p_{k}}\right] \\
& \times\left[\begin{array}{c}
\left(\ln \frac{\tilde{u}_{1}(\omega)}{\tilde{d}_{1}(\omega)}\right)^{*} \\
\tilde{u}_{1}(\omega) \\
0 \\
\vdots \\
0
\end{array}\right] d \omega .
\end{aligned}
$$

The partial-derivative wavefields in equation 15 can be calculated numerically by taking the derivative of the matrix equation resulting from finite-element or finite-difference implementations of the wave equation (see Pratt et al., 1998; Shin et al., 2003). Taking the derivative of equation 3 with respect to parameter $p_{k}$, we obtain

$$
\frac{\partial \mathbf{S}(\omega)}{\partial p_{k}} \widetilde{\mathbf{u}}(\omega)+\mathbf{S}(\omega) \frac{\partial \widetilde{\mathbf{u}}(\omega)}{\partial p_{k}}=\mathbf{0} .
$$

Because the source-related terms $\mathbf{f}(z)$ and $\exp \left[i \theta_{s}^{m}(\omega)\right]$ in equation 3 are not a function of the subsurface parameters, the derivative of 
the source vector with respect to the subsurface parameter becomes zero. From equation 16, we obtain

$$
\frac{\partial \widetilde{\mathbf{u}}(\omega)}{\partial p_{k}}=\mathbf{S}^{-1}(\omega) \mathbf{v}_{k}(\omega)
$$

with

$$
\mathbf{v}_{k}(\omega)=-\frac{\partial \mathbf{S}(\omega)}{\partial p_{k}} \widetilde{\mathbf{u}}(\omega),
$$

where $\mathbf{v}_{k}(\omega)$ is the virtual-source vector, which can be used to compute the partial-derivative wavefield with respect to the $k$ th velocity $p_{k}$.

The partial-derivative wavefield and the virtual source do not exist in reality. Once we factor a complex impedance matrix, we can compute the partial-derivative wavefields for multiple shots as well, using the factored matrix (Pratt et al., 1998). If we introduce the relationship between the virtual source and the partial-derivative wavefield (e.g., equations 17 and 18) into equation 15, the steepest-descent direction at the $k$ th depth point can be expressed as

$$
\phi_{k}=\operatorname{Re}\left\{\int_{-\infty}^{\infty} \mathbf{v}_{k}^{T}(\omega)\left[\mathbf{S}^{-1}(\omega)\right]^{T} \mathbf{r}(\omega) d \omega\right\},
$$

and the entire steepest-descent direction is

$$
\phi=\operatorname{Re}\left\{\int_{-\infty}^{\infty} \mathbf{V}^{T}(\omega)\left[\mathbf{S}^{-1}(\omega)\right]^{T} \mathbf{r}(\omega) d \omega\right\},
$$

where

$$
\mathbf{r}(\omega)=\left[\begin{array}{c}
{\left[\ln \frac{\tilde{u}_{1}(\omega)}{\tilde{d}_{1}(\omega)}\right]^{*}} \\
\tilde{u}_{1}(\omega) \\
0 \\
\vdots \\
0
\end{array}\right]
$$

and $\mathbf{V}$ is the virtual-source matrix whose column is the virtualsource vector $\mathbf{v}_{\mathbf{k}}$ at the $k$ th depth point:

$$
\mathbf{V}=\left[\begin{array}{llll}
\mathbf{v}_{1} & \mathbf{v}_{2} & \cdots & \mathbf{v}_{N}
\end{array}\right]
$$

Because the modeling operator $\mathbf{S}(\omega)$ is self-adjoint, $\left[\mathbf{S}^{-1}(\omega)\right]^{T}$ is interpreted as a back-propagation operator, and consequently the numerical structure of equation 20 is similar to that of reverse-time migration (Pratt et al., 1998; Shin et al., 2003). However, unlike reverse-time migration in which the field data are back-propagated, we take the logarithmic residuals, divide them by the current model response at the surface, and propagate them in backward time. We obtain the steepest-descent direction by calculating the zerolag value of convolution between the back-propagated wavefield and the virtual source, as we normally do in prestack reverse-time migration and prestack waveform inversion (Pratt et al., 1998).
When we compute the steepest-descent direction of the objective function of the amplitude and the phase of the wavefield for the inversion, we can divide the objective function into three types. First, we may take the $l_{2}$ norm of errors of the logarithmic amplitudes and the phases of the wavefields as shown in equation 10 and compute the steepest-descent direction of the objective function using equation 20 . Second, we may take the $l_{2}$ norm of errors of the logarithmic amplitudes of the wavefields and neglect the $l_{2}$ norm of the phase errors in equation 10 . In this case, the steepest-descent direction is expressed by equation 20 with $\mathbf{r}(\omega)$ changed to

$$
\mathbf{r}(\omega)=\left[\begin{array}{c}
\operatorname{Re}\left\{\ln \frac{\tilde{u}_{1}(\omega)}{\tilde{d}_{1}(\omega)}\right\} \\
\tilde{u}_{1}(\omega) \\
0 \\
\vdots \\
0
\end{array}\right] .
$$

Third, we may take the $l_{2}$ norm of the phase residuals by discarding the $l_{2}$ norm of the logarithmic amplitude errors in equation 10 . For computing the steepest-descent direction, we change the residual vector $\mathbf{r}(\omega)$ of equation 20 to

$$
\mathbf{r}(\omega)=\left[\begin{array}{c}
-i \operatorname{Im}\left\{\ln \frac{\tilde{u}_{1}(\omega)}{\tilde{d}_{1}(\omega)}\right\} \\
\tilde{u}_{1}(\omega) \\
0 \\
\vdots \\
0
\end{array}\right] .
$$

\section{ESTIMATION OF SOURCE WAVELET}

From equation 10, we note that the objective function is a function of the phase and amplitude of the source wavelet. Applying the full Newton method (Lines and Treitel, 1984) to estimate the phase and amplitude of the source signature, we compute

$$
\delta \mathbf{p}_{s}=-\mathbf{H}_{s}^{-1} \nabla_{s} E
$$

where $\delta \mathbf{p}_{s}$ is a vector containing the change in phase and amplitude of the source signature and $\nabla_{s} E$ and $\mathbf{H}_{s}$ are the gradient and the Hessian of the objective function with respect to amplitude and phase of the source wavelet, respectively. Because there is no relationship between phase and amplitude of the source wavelet in the objective function, as given in equation 10, the mixed, partial derivatives with respect to phase and amplitude of the source wavelet become zero, resulting in a diagonal Hessian matrix. The first derivative of equation 10 with respect to the phase of the source wavelet is

$$
\frac{\partial E}{\partial \theta_{s}(\omega)}=\left[\theta_{1}^{m}(\omega)+\theta_{s}(\omega)-\theta_{1}^{f}(\omega)\right]
$$

and the second derivative is 


$$
\frac{\partial^{2} E}{\partial \theta_{s}^{2}(\omega)}=1
$$

Note that the first derivative is in fact the phase error, whereas the second derivative degenerates to a constant. In the inversion process, we obtain the phase error at each frequency and then use it to update the source wavelet phase. At each iteration, we update the phase of the source function in equation 3 with

$$
\theta_{s}^{(k+1)}(\omega)=\theta_{s}^{(k)}(\omega)+\left[\theta_{1}^{m}(\omega)+\theta_{s}(\omega)-\theta_{1}^{f}(\omega)\right]^{(k)},
$$

where $k$ indicates the iteration number.

In a similar manner, we obtain the first and second derivatives of the objective function with respect to amplitude of the source signature as

$$
\frac{\partial E}{\partial g_{s}(\omega)}=\frac{1}{g_{s}(\omega)} \ln \frac{A_{1}^{m}(\omega) g_{s}(\omega)}{A_{1}^{f}(\omega)}
$$

and

$$
\frac{\partial^{2} E}{\partial g_{s}^{2}(\omega)}=\frac{1}{g_{s}(\omega)^{2}}\left(1-\ln \frac{A_{1}^{m}(\omega) g_{s}(\omega)}{A_{1}^{f}(\omega)}\right) .
$$

Therefore, we update the source-function amplitude at each iteration with

$$
g_{s}^{(k+1)}(\omega)=g_{s}^{(k)}(\omega) \frac{1-2 \ln \frac{A_{1}^{m}(\omega) g_{s}^{(k)}(\omega)}{A_{1}^{f}(\omega)}}{1-\ln \frac{A_{1}^{m}(\omega) g_{s}^{(k)}(\omega)}{A_{1}^{f}(\omega)}}
$$

In the case of multiple receivers, equations 28 and 31 can be expressed as

$$
\begin{aligned}
\theta_{s}^{(k+1)}(\omega)= & \theta_{s}^{(k)}(\omega)+\frac{1}{n_{s} n_{r}} \sum_{i=1}^{n_{s}} \sum_{j=1}^{n_{r}}\left[\theta_{i j}^{m}(\omega)+\theta_{s}(\omega)\right. \\
& \left.-\theta_{i j}^{f}(\omega)\right]^{(k)}
\end{aligned}
$$

and

$$
g_{s}^{(k+1)}(\omega)=g_{s}^{(k)}(\omega) \frac{1-\frac{2}{n_{s} n_{r}} \sum_{i=1}^{n_{s}} \sum_{j=1}^{n_{r}} \ln \frac{A_{i j}^{m}(\omega) g_{s}^{(k)}(\omega)}{A_{i j}^{f}(\omega)}}{1-\frac{1}{n_{s} n_{r}} \sum_{i=1}^{n_{s}} \sum_{j=1}^{n_{r}} \ln \frac{A_{i j}^{m}(\omega) g_{s}^{(k)}(\omega)}{A_{i j}^{f}(\omega)}},
$$

respectively, where $i$ and $j$ indicate the source and receiver numbers. Based on the above results, we may iteratively update phase, amplitude, or both phase and amplitude of the source wavelet during the waveform-inversion process.

\section{Regularization of the steepest-descent direction}

In order to enhance the stability of our inversion algorithm, we need to regularize the steepest-descent direction. The approximate Hessian matrix in the Gauss-Newton method is a frequent method of choice for regularization of inversion algorithms, but its computation along with its inverse are usually very expensive. In our algorithm, we use a pseudo-Hessian matrix for regularization in order to achieve computational efficiency (Shin et al., 2001a).

When we apply the Gauss-Newton method to the frequencydomain seismic inverse problem, the normal equation can be expressed as

$$
-\phi=\operatorname{Re}\left\{\int_{-\infty}^{\infty} \mathbf{J}^{* T}(\omega) \mathbf{J}(\omega) d \omega\right\} \delta \mathbf{p}
$$

with

$$
\mathbf{J}(\omega)=\left[\frac{1}{\tilde{u}_{1}} \frac{\partial \tilde{u}_{1}(\omega)}{\partial p_{1}} \frac{1}{\tilde{u}_{1}} \frac{\partial \tilde{u}_{1}(\omega)}{\partial p_{2}} \frac{1}{\tilde{u}_{1}} \frac{\partial \tilde{u}_{1}(\omega)}{\partial p_{3}} \cdots\right]
$$

where $\mathbf{J}^{* \mathbf{T}} \mathbf{J}$ is the $n \times n$ approximate Hessian matrix (Shin, 1988).

Even if we use the reciprocity theorem to compute the partialderivative wavefield (Shin et al., 2001b), it is difficult to calculate explicitly the approximate Hessian matrix for large-scale 2D or 3D velocity models. For example, consider the parametrization of the $2 \mathrm{D}$ velocity model into $2000 \times 1000$ unknowns: The size of the approximate Hessian matrix is $2000000 \times 2000000$ for each source. Consequently, the computation of this matrix inverse is beyond the capability of current computers. Another problem related to the Hessian matrix for seismic inverse problems is that this matrix is ill-posed. For most seismic inverse problems, the approximate Hessian matrix is not invertible, because some of its diagonal elements have small values, close to zero, which originate from poorly illuminated areas and geometric spreading (Chavent and Plessix, 1999). To overcome this, Shin et al. (2001a) suggested replacing the approximate Hessian matrix with the pseudo-Hessian matrix. The main idea was to use the similarity between the uncorrelated structure of the forward-modeled wavefields and the uncorrelated property of the partial-derivative wavefields.

We use the approach of Shin et al. (2001a) in place of the approximate Hessian matrix for regularization of the steepest-descent direction. The main goal is to amplify the small values of the steepest-descent direction caused by geometric spreading. We accomplish this goal by multiplying the steepest-descent direction by the reciprocal of the diagonal elements of the pseudo-Hessian matrix. In the frequency domain, we have two choices for regularization of the steepest-descent direction. One choice is to take an average value of step lengths, obtained by dividing the steepestdescent direction by the diagonal elements of the pseudo-Hessian matrix at each frequency. The other choice is to sum the steepestdescent direction at all frequencies and the pseudo-Hessian matrix at all frequencies separately and then divide the summed steepestdescent direction by the summed pseudo-Hessian matrix. In order to avoid the singularity problem of the pseudo-Hessian matrix when using the average step-length approach, we also use a Lagrangian multiplier. This can be expressed as 


$$
\begin{aligned}
\delta \mathbf{p}= & -\operatorname{Re}\left\{\int_{-\infty}^{\infty}\left[\operatorname{diag}\left\{\operatorname{Re}\left(\mathbf{V}^{*} \mathbf{T}(\omega) \mathbf{V}(\omega)\right)+\lambda \mathbf{I}\right\}\right]^{-1}\right. \\
& \left.\times \mathbf{V}^{\mathbf{T}}\left[\mathbf{S}^{-1}(\omega)\right]^{\mathbf{T}} \mathbf{r}(\omega) \mathrm{d} \omega\right\}
\end{aligned}
$$

where $\lambda$ is the damping factor. For the damping factor in our inversion, we choose either $0.1 \%, 1 \%$, or $2 \%$ of the maximum value of the diagonal elements in the pseudo-Hessian matrix.

\section{NUMERICAL EXAMPLES}

\section{Synthetic data without noise}

We first test our waveform-inversion algorithms on synthetic data generated for the Institute Français du Pétrole (IFP) Marmousi model (Versteeg, 1994). Figure 1 shows the 16-m-grid Marmousi model. To generate synthetic seismograms from it, we used the nine-point, frequency-domain, finite-difference modeling technique suggested by Jo et al. (1996). We use the first derivative of a Gaussian function with maximum frequency of $18.41 \mathrm{~Hz}$ for the source wavelet and perform the inversion for 59 frequencies, ranging from $0.3121 \mathrm{~Hz}$ to $18.41 \mathrm{~Hz}$. By distributing the inversion at each frequency to different processors using the Message Passing Interference (MPI) system, we gain computational efficiency. We locate 576 shots at the surface at an interval of $16 \mathrm{~m}$. In all the cases, we use the full aperture of the model, locating 576 receivers on all nodal points at the surface. Figure 2 shows an example seismogram generated by the nine-point, frequency-domain, finite-difference method for the Marmousi model, when the source is located at $3200 \mathrm{~m}$.

We first perform full-waveform inversion employing both amplitude and phase based on equations 20 and 21. For an initial model for the inversion, we take a linearly increasing velocity model, where velocity varies from $1500 \mathrm{~m} / \mathrm{s}$ to $4500 \mathrm{~m} / \mathrm{s}$ as shown in Figure 3a. In Figure 3b, we present the velocity structure obtained by our full-waveform inversion algorithm at the 1928th iteration. Because we use small step lengths, the convergence rate is very slow. We could accelerate convergence using other optimization techniques, such as the conjugate-gradient method. In the inversion, we update the source wavelet's amplitude and phase at each iteration. In order to check the accuracy of our waveforminversion algorithm, we compare the velocity model inverted by our algorithm to that of the conventional, source-independent

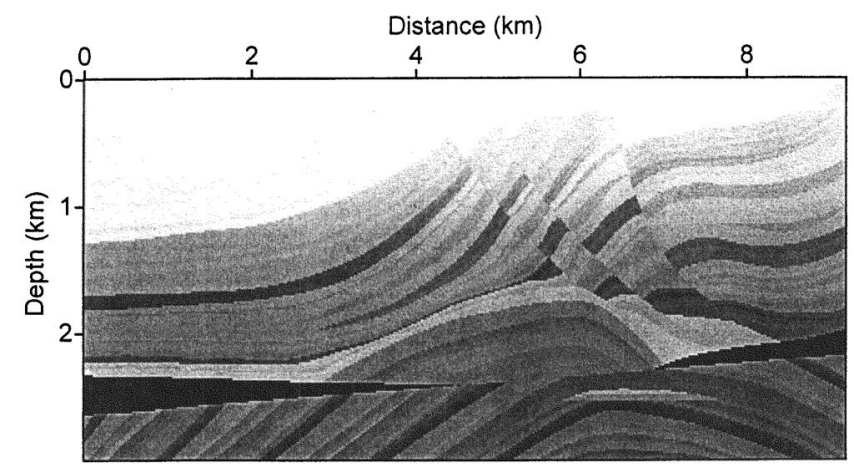

Figure 1. The 2D Institute Français du Pétrole (IFP) Marmousi model. waveform inversion. For the conventional, source-independent inversion algorithm, we construct the objective function using the $l_{2}$ norm of residuals between field data and model responses normalized by the reference wavefields. With the exception of the objective function, the numerical algorithm for conventional inversion is the same as ours. Figure $3 \mathrm{c}$ shows the velocity structure generated by the conventional inversion algorithm. In Figure 3c, the left part of the inverted model is not accurately recovered. For quantitative comparison, we measure the misfit of the velocities obtained by the two inversion algorithms with respect to the true velocities (Figure $4 \mathrm{a}$ and $\mathrm{b}$ ). From Figure $4 \mathrm{a}$ and $\mathrm{b}$, we note that our waveforminversion algorithm gives more reliable results than the conventional method does.

We also need to check whether the source wavelet is properly inverted. In Figure 5, we display amplitude and phase spectra of the true- and estimated-source signature with respect to frequency. In Figure 5, the amplitudes of the estimated source are consistent with those of the true-source wavelet, but the phase spectrum shows some discrepancies at the frequencies where phases are wrapped.

In order to measure the sensitivity of the inversion results to amplitude and phase, we also show velocity models inverted by the pure-amplitude method (equations 20 and 23) at the 198th iteration and the pure-phase method (equations 20 and 24) at the 1253rd iteration in Figure 6a and b, respectively. In each case, we invert either amplitude or phase of the source signature. For an initial model, we choose the linearly increasing velocity model (Figure $3 a)$. From Figure $6 a$ and $b$, we note that the pure-phase method gives a velocity model comparable to that of the full waveforminversion method using both amplitude and phase, whereas the pure amplitude method does not yield good results. Figure 7 shows rms errors of amplitude and phase with respect to iteration. While the rms error of amplitude rapidly converges to zero, the rms error of phase decreases monotonically as iteration number increases.

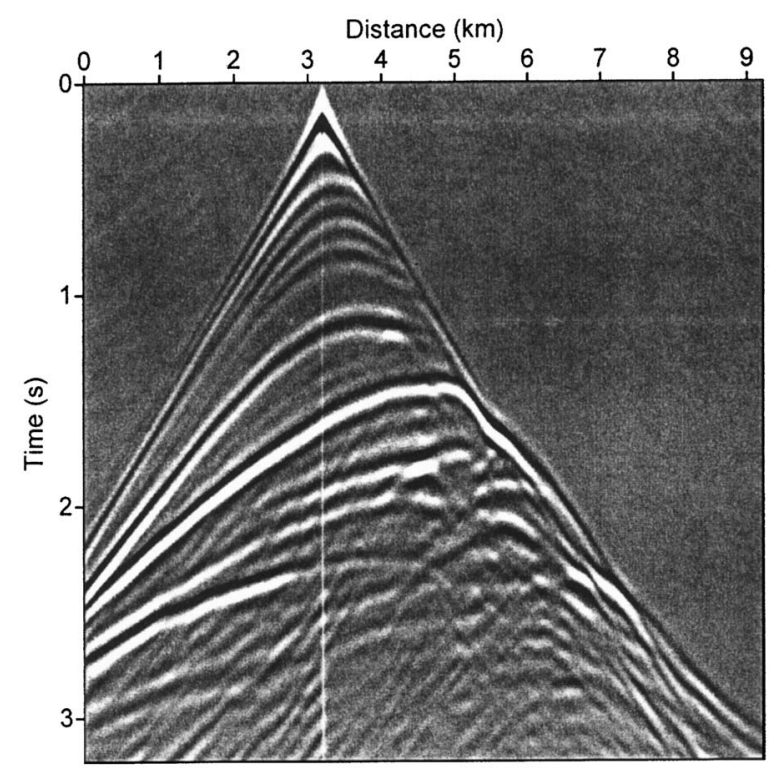

Figure 2. The synthetic seismograms generated by the nine-point, frequency-domain, finite-difference method for the Marmousi model. 


\section{Synthetic data with noise}

Before applying our inversion to real seismic data, which is usually contaminated with noise, we perform the inversion on the Marmousi synthetic data set with random noise added to it. The average level of random noise is about $25 \%$ of the original amplitude at each frequency. Figure 8 shows the noise-added shot gather when the source is located at $3200 \mathrm{~m}$ (compare Figure 8 with Figure 2). Figure 9 shows the velocity model inverted by our waveform-inversion technique at the 926th iteration for the noise-added data. The velocity model inverted for the noise-added data is very similar to that for the noise-free data (compare Figure 9 with Figure $3 \mathrm{~b}$ ). In Figure 10, we check the source-signature estimate by comparing the spectra of the estimated-source wavelet with those of true-source wavelet. The estimated amplitude and phase are comparable to those of the true wavelet, although slightly more differences exist than in the noise-free examples.

a)

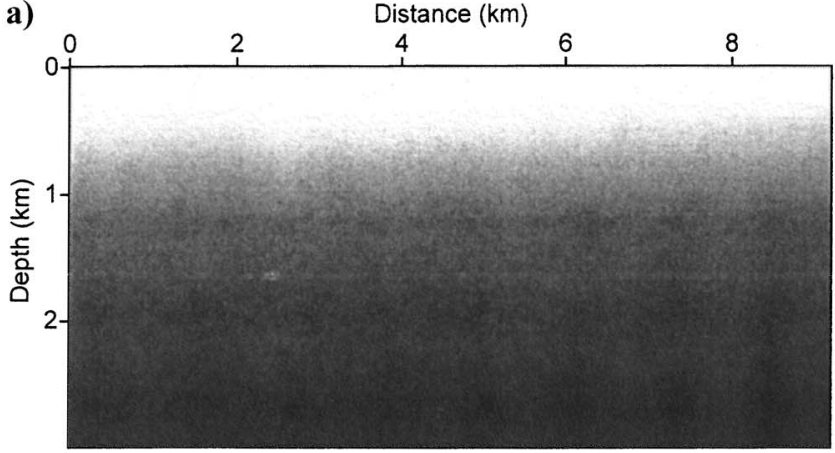

b)

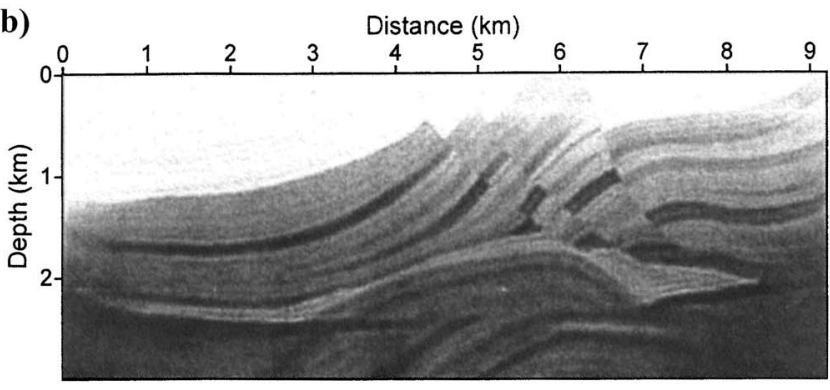

c)

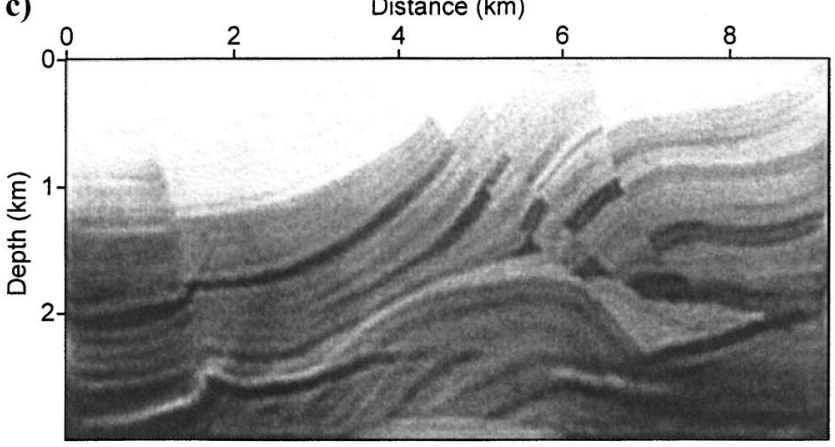

Figure 3. Numerical examples for the noise-free Marmousi synthetic data: (a) the initial model, (b) the velocity model inverted by full waveform inversion using both amplitude and phase at the 1928th iteration, and (c) the velocity model inverted by the conventional source-independent inversion algorithm at the 259th iteration.
In real seismic data, noise is often dominant in the low- and high-frequency bands. In our frequency-domain waveform inversion, we use only relatively low frequencies because of the lack of computational resources; therefore, we consider noise effects only
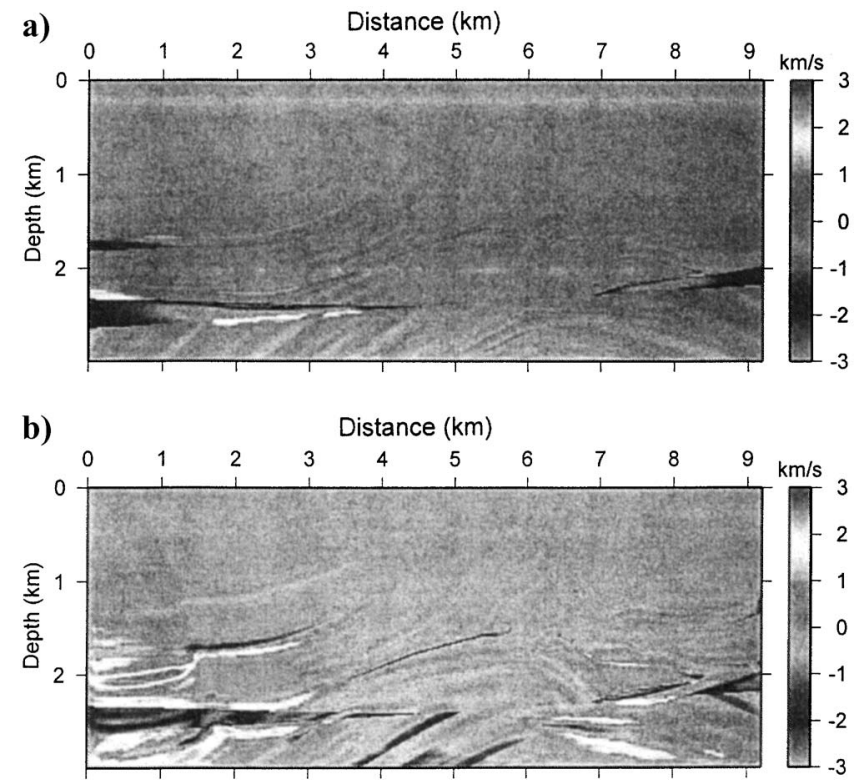

Figure 4. Misfits of velocity models generated for the noise-free Marmousi synthetic data by (a) our inversion algorithm and (b) the conventional source-independent inversion algorithm with respect to the true Marmousi model.
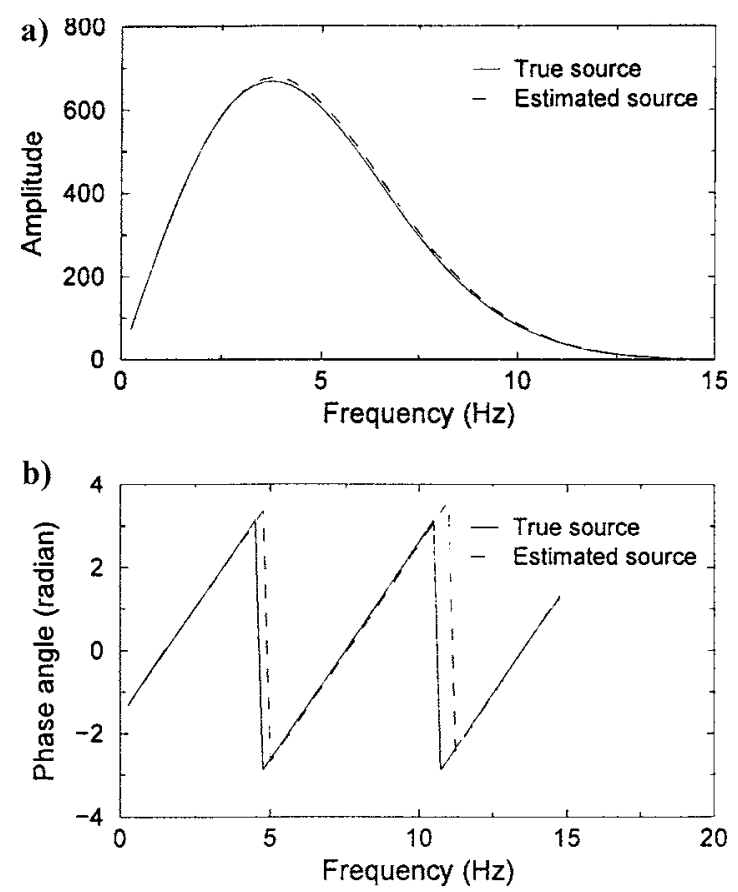

Figure 5. Spectra of (a) amplitude and (b) phase of the true-source signature and the estimated-source signature for the noise-free Marmousi synthetic data. The solid line represents the true-source signature, and the dashed line denotes the estimated-source signature. 
in the low-frequency band. To do so, we apply our full waveforminversion method to the noise-free synthetic data set without frequencies below $5 \mathrm{~Hz}$. In Figures 11 and 12, we display the inversion results for the velocity model and source wavelet. The in-
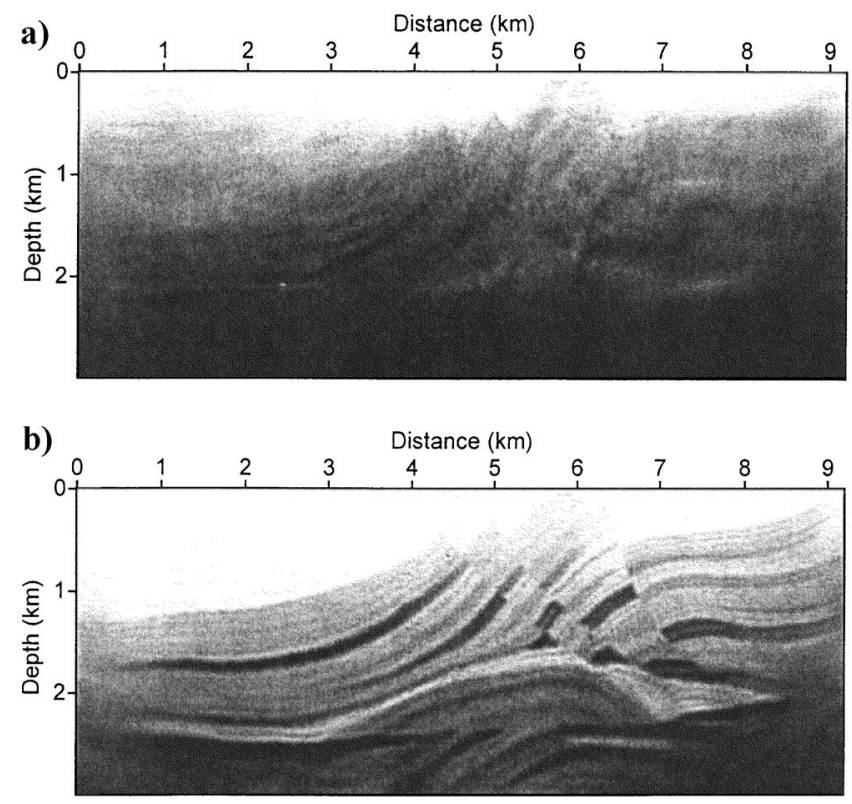

Figure 6. Velocity structures generated for the noise-free Marmousi synthetic data by (a) the pure amplitude inversion method at the 198th iteration and (b) the pure phase inversion method at the 1253rd iteration.
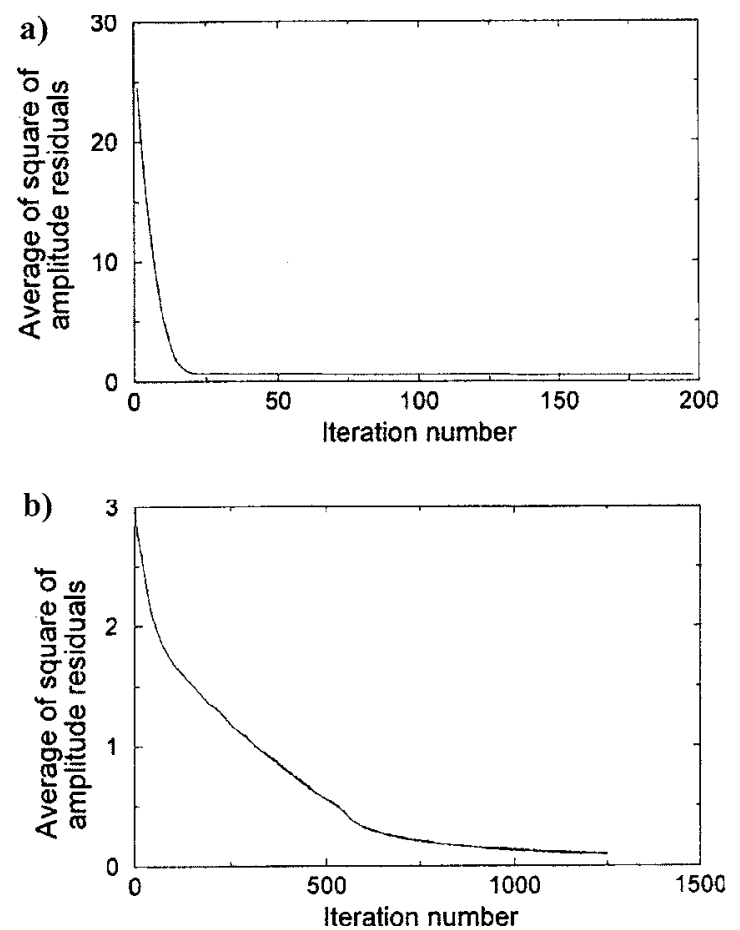

Figure 7. The history of errors of (a) the pure amplitude inversion and (b) the pure phase inversion algorithm for the noise-free Marmousi synthetic data. verted velocity model and source-wavelet spectra for the lowfrequency missing data are not as good as they are for the random noise-added data (Figures 9 and 10). We note a large discrepancy in the phase spectrum of Figure 12. These results confirm the fact that low-frequency data are of critical importance in waveform inversion (Lines, personal communication, 1987; Song et al., 1995).

\section{Field data}

After successfully completing the experiment on the synthetic data from the Marmousi model, we applied our full waveforminversion algorithm to real data gathered from the Korean continental shelf, where gas deposits exist. The field data were collected with 120 channels for 235 shots. The shot interval and receiver interval are $25 \mathrm{~m}$, and the time sample interval is $4 \mathrm{~ms}$. A receivercable length of $2975 \mathrm{~m}$ resulted in minimum and maximum offset distances of $300 \mathrm{~m}$ and $3285 \mathrm{~m}$, respectively. Figure 13 shows a representative common-shot gather, where data are muted to the temporal range containing reflections/refractions from a gas reservoir. We use low-pass filtered data from $0.5 \mathrm{~Hz}$ to $24 \mathrm{~Hz}$ and a finite-difference grid interval of $12.5 \mathrm{~m}$ to prevent grid dispersion.

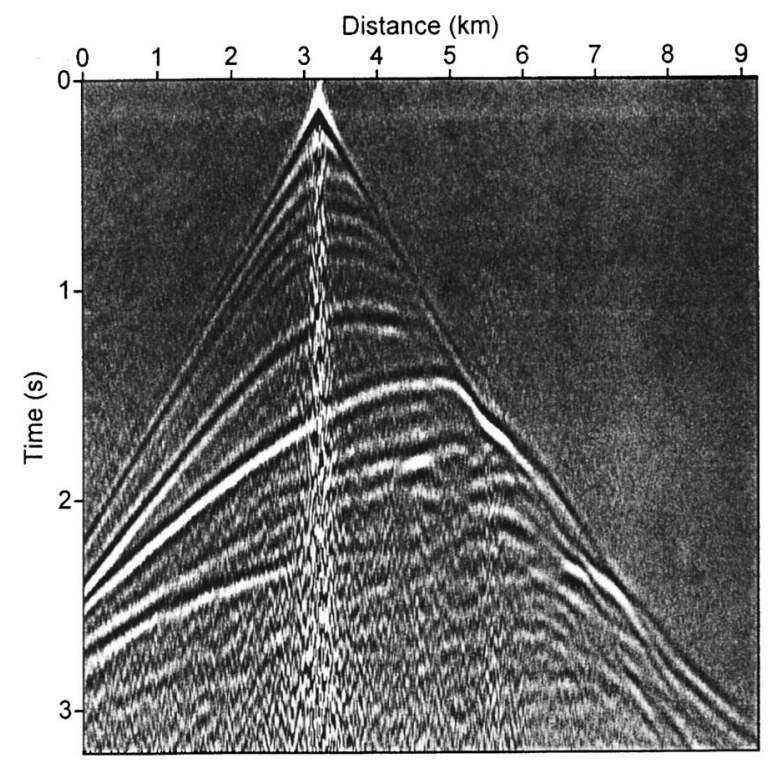

Figure 8 . The synthetic seismograms with random noise generated by the nine-point, frequency-domain, finite-difference method for the Marmousi model. The noise level is $25 \%$.

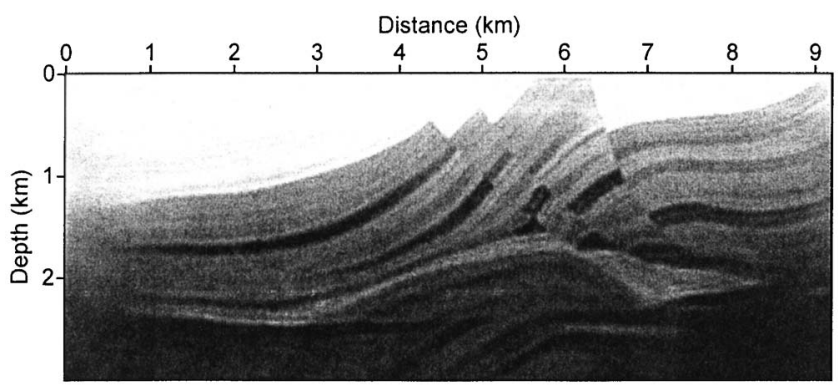

Figure 9. The velocity model inverted by the full waveform inversion using both amplitude and phase at the 926th iteration for the random noise-added Marmousi synthetic data. 
For an initial model for the real-data inversion, we chose a linearly increasing velocity model (Figure 14a), as we did for the synthetic Marmousi data. Of the three types of inversion algorithms, we apply only the full waveform-inversion algorithm for inverting both the real data and the source signature up to $24 \mathrm{~Hz}$. In the realdata inversion, we update the velocity model by inverting real data for each frequency, summing the results at every frequency and then computing the steepest-descent direction.

In Figure 14b, which shows the inverted velocity model at the 252nd iteration, we can see the shallow, stratified layers and the area (denoted by an arrow) of a gas reservoir. However, Figure 14b also reveals that the inverted velocity model is not far from the initial velocity model, which indicates that we could not fully recover the velocity model's long-wavelength content because of the real data's unreliable low-frequency content. Figure 15 shows the rms errors of amplitudes and phases with respect to iteration number, respectively. As in the synthetic examples, the rms error of amplitude converges more rapidly than that of phase.

We check the validity of the inverted model by comparing the
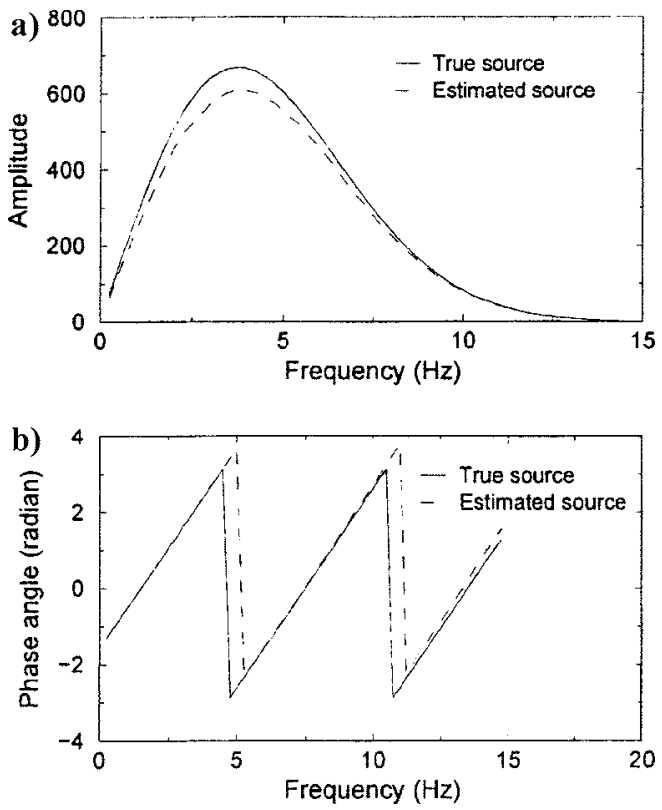

Figure 10. Spectra of (a) amplitude and (b) phase of the true- and the estimated-source signature for the random noise-added data. The solid line represents the true-source signature, and the dashed line denotes the estimated-source signature.

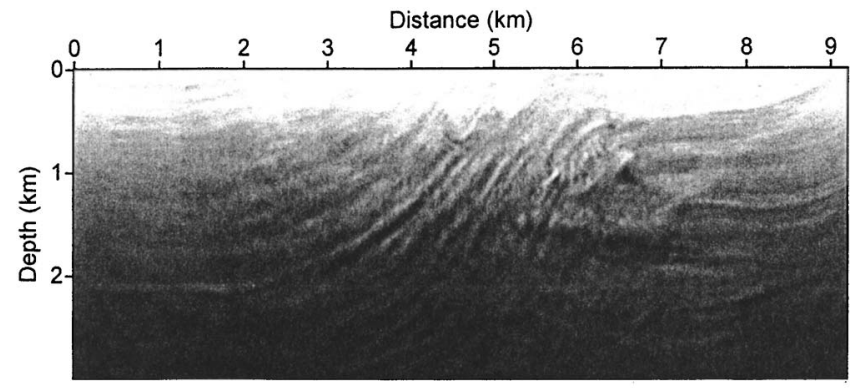

Figure 11 . The velocity model inverted by our full waveform inversion at the 363rd iteration for the Marmousi synthetic data without frequencies lower than $5 \mathrm{~Hz}$. real seismogram with the synthetic seismogram generated for the inverted model shown in Figure 16. In Figure 16, we display the real seismogram low-pass filtered up to $24 \mathrm{~Hz}$ and the synthetic seismogram when a source is located at $1.375 \mathrm{~km}$. The synthetic seismogram generated from the inverted model resembles the real seismogram. Figure 17 shows single traces of the real and synthetic seismograms. The single traces are obtained at three receivers separated by $2.375 \mathrm{~km}, 3.0 \mathrm{~km}$, and $3.625 \mathrm{~km}$ from the source. In Figure 17, we see that although there are some discrepancies, the synthetic data are comparable to the field data. Possible sources of discrepancies are the incompletely inverted velocity model because of the unreliable low-frequency content of real data, a local minimum problem and a nonuniqueness problem in a general in-
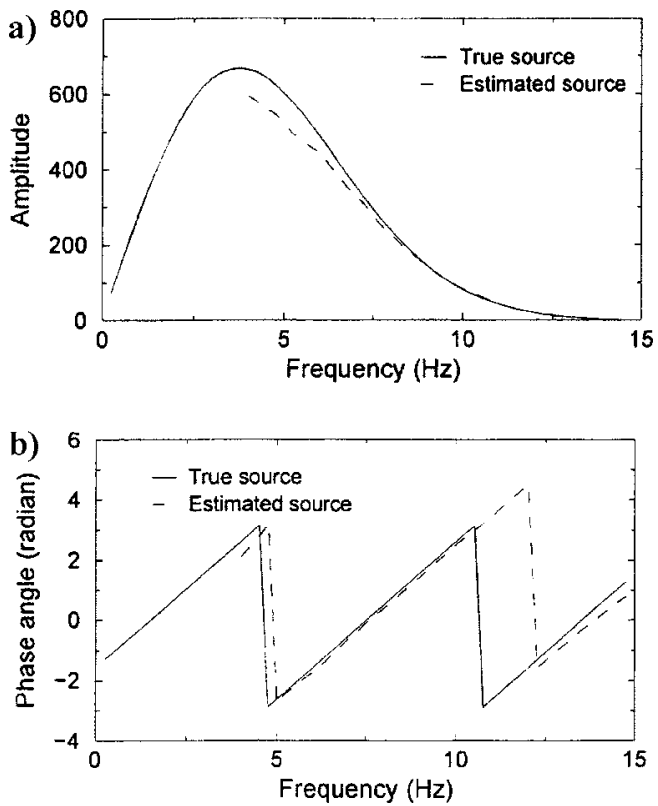

Figure 12. Spectra of (a) amplitude and (b) phase of the true- and the estimated-source signature for the data without frequencies below $5 \mathrm{~Hz}$. The solid line represents the true-source signature, and the dashed line denotes the estimated-source signature.

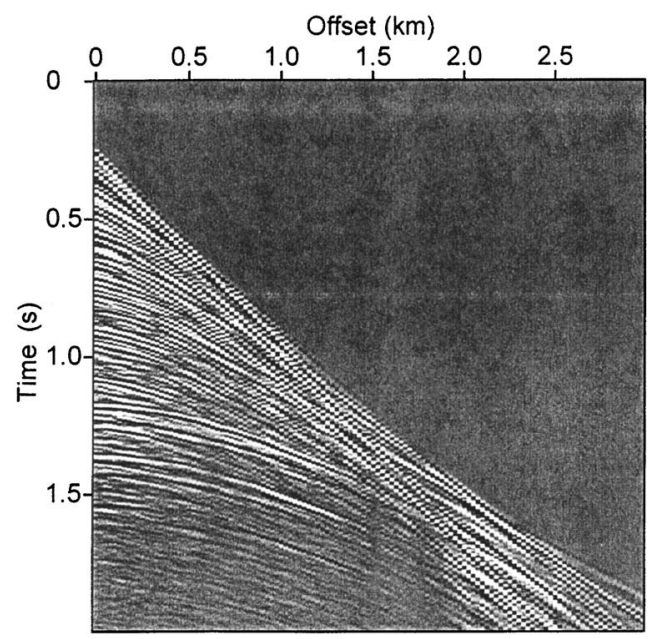

Figure 13. Real seismogram collected on the Korean continental shelf. 
verse problem, the inadequacy of the acoustic approximation to 3D real-wave propagation, array forming of hydrophones in the streamer cable, nonlinear wave propagation near a source point, a
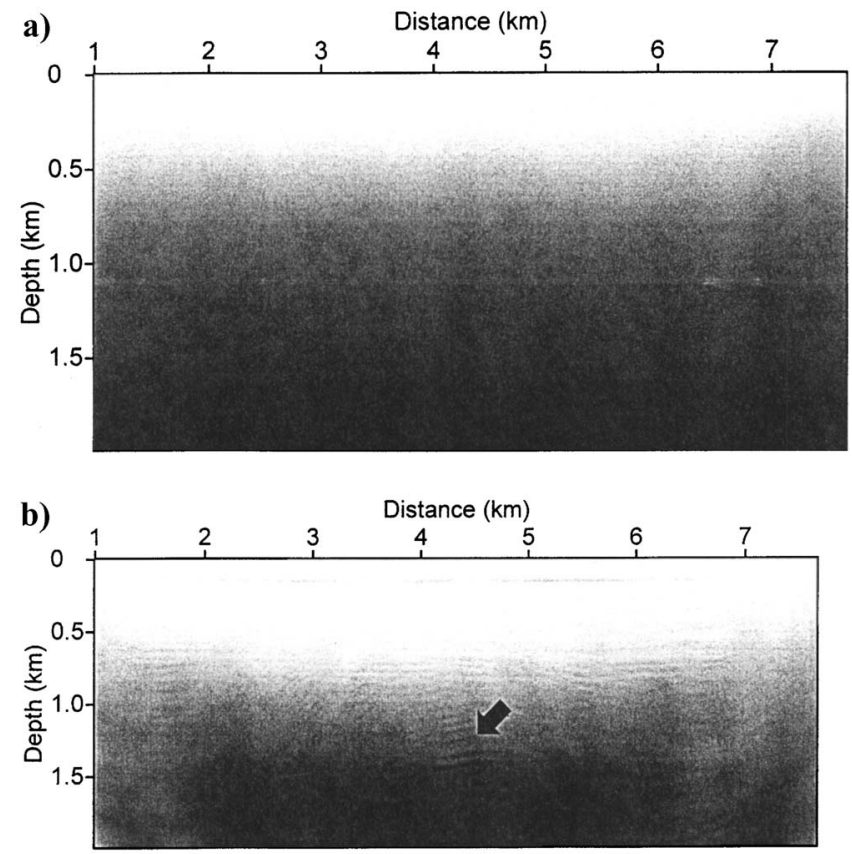

Figure 14. Real data examples: (a) the initial model used for waveform inversion of the field data, and (b) the inverted velocity structure of the field data collected on the Korean continental shelf at the 252nd iteration.
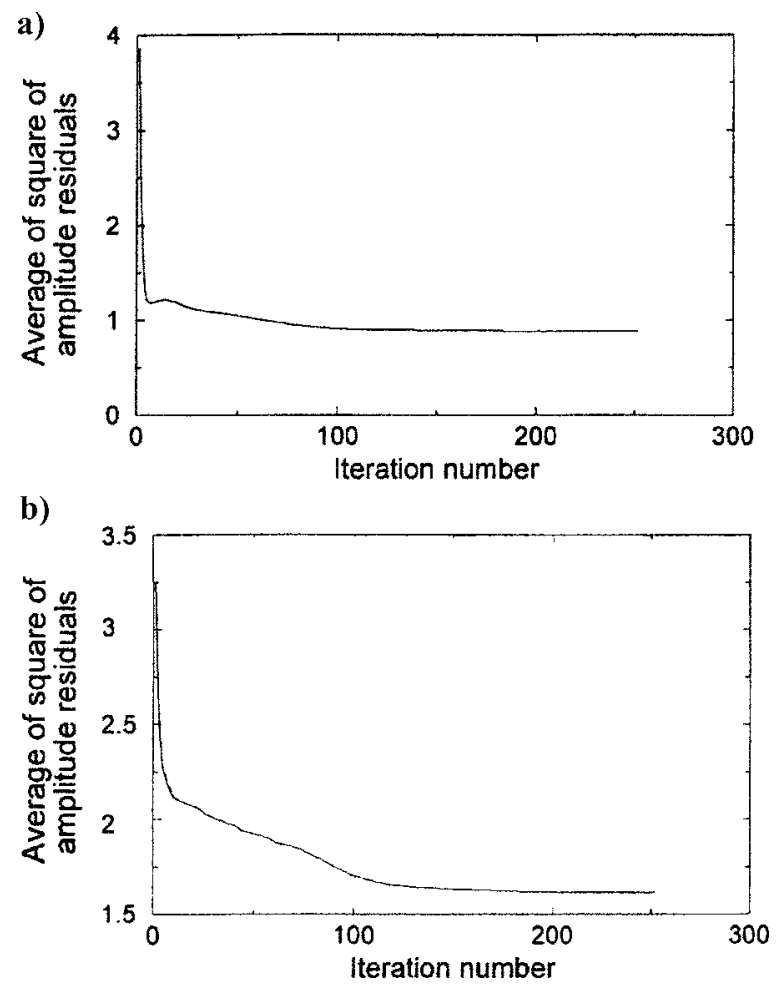

Figure 15. History of (a) amplitude and (b) phase errors of the real seismogram collected on the Korean continental shelf.
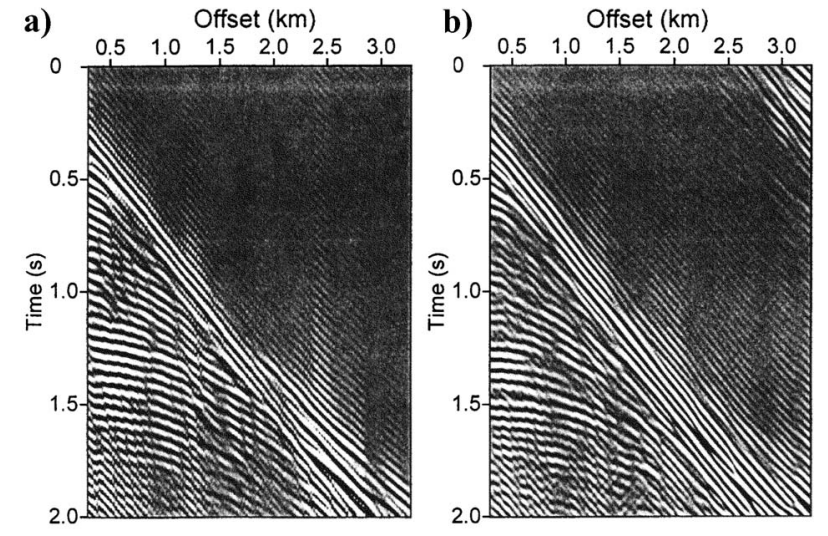

Figure 16. Comparison of (a) field seismogram with (b) synthetic seismogram generated from the inverted velocity structure shown in Figure $14 \mathrm{~b}$ when a source is located at $1.375 \mathrm{~km}$.
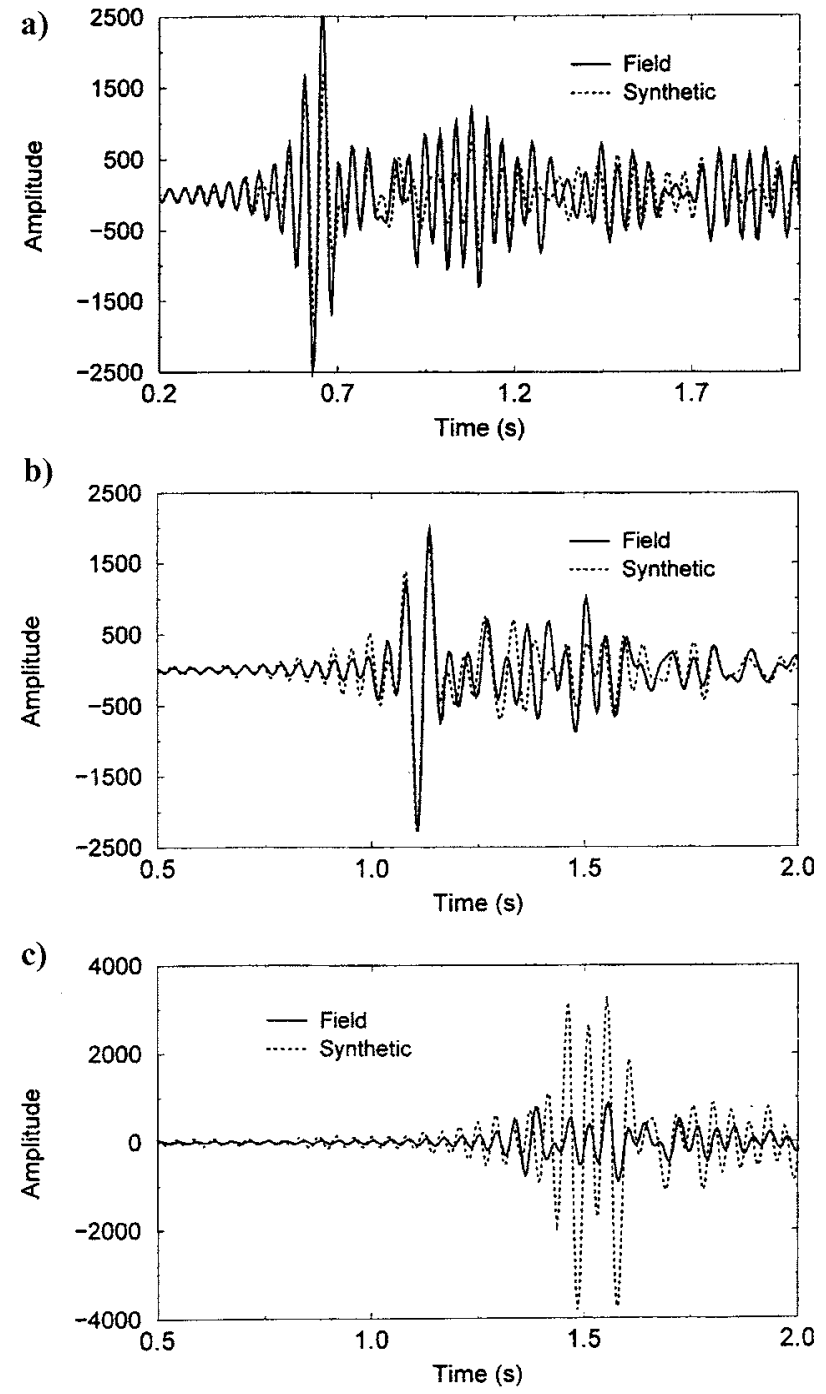

Figure 17. Comparison of single traces of field data with those of synthetic data obtained from the inverted velocity structure (e.g., Figure 14b). Receivers are located at (a) $2.375 \mathrm{~km}$, (b) $3 \mathrm{~km}$, and (c) $3.625 \mathrm{~km}$ from the source. The solid line indicates the field data, and the dotted line denotes the synthetic data. 
problem of source-receiver coupling to the earth, 2D approximation to 3D wavefields, and anisotropic properties and intrinsic attenuation of the real earth.

\section{CONCLUSIONS}

Instead of defining the objective function as the $l_{2}$ norm of residuals between the measured data and the modeled data, we built an objective function that can be separated into three types via the complex phase: the mismatch of amplitude, the mismatch of phase, and the mismatch of both amplitude and phase. This separation leads to three kinds of inversions: the simultaneous inversion of amplitude and phase, pure-amplitude inversion, or pure-phase inversion. We estimated the source signature as well as the subsurface velocity by including amplitude and phase of the source signature in our objective function.

In our waveform inversion, we computed the steepest-descent direction of the three different objective functions without directly computing the sensitivity matrix. The steepest-descent direction is computed by the back-propagation algorithm of reverse-time migration on the basis of the matrix formalism of the frequencydomain, finite-element/finite-difference method for the wave equation. Our algorithm shares the same numerical algorithm with prestack reverse-time migration and seismic inversion. Although the conventional waveform inversion using the back-propagation technique back-propagates the residuals between the measured and the modeled data, our approach back-propagates the wavefield obtained by dividing the logarithm of the ratio of the modeled data to the measured data by the modeled wavefield.

By applying three inversion algorithms to the synthetic seismogram of the Marmousi model, we showed that the simultaneous inversion of amplitude and phase produces the best resolution in the shallow and the deep structure of the Marmousi model. Nevertheless, it is too early to state with certainty which inversion algorithm yields the best results because we used only data containing relatively low frequencies ranging from $0.3121 \mathrm{~Hz}$ to $18.41 \mathrm{~Hz}$. We also tested our algorithm for two cases of corrupted Marmousi synthetic data. One test involved inversion of data with random noise added, and the other involved inversion of data without frequencies below $5 \mathrm{~Hz}$. For the former, the inverted velocity model was as close as that of the noise-free data. For the latter, the inversion results were not good, which indicates that our logarithmic-waveform inversion cannot resolve low wavenumbers of the velocity model, a situation like conventional waveform inversion when low frequencies are missing.

By applying our inversion algorithm to real seismic data, we converged to a velocity structure. However, we are still uncertain whether our inversion algorithm can be applied to real seismic data, when low-frequency components are often missing. Moreover, in waveform inversion of real data, we also need to overcome intrinsic problems such as the incompletely inverted velocity model arising from the unreliable low-frequency content of real data, a local minimum problem and nonuniqueness of the general inverse problem, the inadequacy of the acoustic approximation to 3D real-wave propagation, array forming of hydrophones in the streamer cable, nonlinear wave propagation near a source point, a source-receiver coupling problem, 2D approximation to 3D wavefields, and anisotropic properties and attenuation of the real earth.

In our waveform inversion, we employed a small step length, resulting in slow convergence, but the convergence can be acceler- ated by using methods such as conjugate gradient. We also used wrapped phases rather than the more meaningful unwrapped phases, which may sacrifice something unknown in inverting the velocity structures.

For a reliable inversion algorithm, we need to unwrap the wrapped phases. Our algorithm can be extended to 2D elasticwaveform inversion and 3D acoustic- and elastic-waveform inversion. Extending our algorithm to the Gauss-Newton method using the reciprocity theorem is straightforward but is computationally more expensive.

\section{ACKNOWLEDGMENTS}

This work was financially supported by National Laboratory Project of Ministry of Science and Technology, Brain Korea 21 project of Ministry of Education, and grant numbers PE93300 and PM31600 from Korea Ocean Research and Development Institute. We would like to thank the Korea National Oil Corporation for providing real seismic data. We also express our gratitude to Sukjoon Pyun at Seoul National University, who provided the velocity model inverted by conventional source-independent waveform inversion.

\section{REFERENCES}

Alford, R. M., K. R. Kelly, and D. M. Boore, 1974, Accuracy of finite difference modeling of the acoustic wave equation: Geophysics, 39, 834842

Bamberger, A., G. Chavent, C. Hemon, and P. Lailly, 1982, Inversion of normal incidence seismograms: Geophysics, 46, 757-770.

Cao, D., W. B. Beydoun, S. C. Singh, and A. Tarantola, 1990, A simultaneous inversion for background velocity and impedance maps: Geophysics, 55, 458-469.

Chavent, G., and R. Plessix, 1999, An optimal true-amplitude least squares prestack depth-migration operator: Geophysics, 64, 508-515.

Gauthier, O., J. Virieux, and A. Tarantola, 1986, Two-dimensional nonlinear inversion of seismic waveforms: Numerical results: Geophysics, $\mathbf{5 1}$ 1387-1403.

Hicks, G. J., and R. G. Pratt, 2001, Reflection waveform inversion using local descent methods: Estimating attenuation and velocity over a gas-sand deposit: Geophysics, 66, 598-612.

Jo, C. H., C. Shin, and J. H. Suh, 1996, Design of an optimal 9 point finite difference frequency-space acoustic wave equation scheme for inversion and modeling: Geophysics, 61, 329-337.

Kolb, P., F. Collino, and P. Lailly, 1986, Prestack inversion of a 1D medium: Proceedings of the Institute of Electrical and Electronics Engineers, Inc., 74, 498-506.

Lailly, P., 1983, The seismic inverse problem as a sequence of before stack migration, in J. B. Bednar, R. Rednar, E. Robinson, and A. Weglein, eds., Conference on inverse scattering: Theory and application: Society for Industrial and Applied Mathematics.

Lines, L. R., and S. Treitel, 1984, A review of least-squares inversion and its application to geophysical problems: Geophysical Prospecting, 32, $159-186$.

Marfurt, K. J., 1984, Accuracy of finite-difference and finite-element modeling of the scalar and elastic wave equation: Geophysics, 49, 533-549.

Marfurt, K. J., and C. Shin 1989, The future of iterative modeling of geophysical exploration, in E. Eisner, ed., Supercomputers in seismic exploration: Pergamon Press.

Mora, P., 1987, Nonlinear two-dimensional elastic inversion of multioffset seismic data: Geophysics, 52, 1211-1228.

- 1989, Inversion $=$ migration + tomography: Geophysics, $\mathbf{5 4}$, $1575-1586$

Pica, A., J. P. Diet, and A. Tarantola, 1990, Nonlinear inversion of seismic reflection data in a laterally invariant medium: Geophysics, 55, 284292.

Pratt, R. G., 1999, Seismic waveform inversion in the frequency domain, Part1: Theory and verification in a physical scale model: Geophysics, 64, 888-901.

Pratt, R. G., C. Shin, and G. J. Hicks, 1998, Gauss-Newton and full Newton methods in frequency domain seismic waveform inversion: Geophysical Journal International, 133, 341-362. 
Pratt, R. G., and R. M. Shipp, 1999, Seismic waveform inversion in the frequency domain, Part 2: Fault delineation in sediments using cross hole data: Geophysics, 64, 902-914.

Shin, C., 1988, Nonlinear elastic wave inversion by blocky parametrization: Ph.D. thesis, University of Tulsa.

Shin, C., S. H. Jang, and D. J. Min, 2001a, Improved amplitude preservation for prestack depth migration by inverse scattering theory: Geophysical Prospecting, 49, 592-606.

Shin, C., D. J. Min, D. W. Yang, and S. K. Lee, 2003, Evaluation of post stack migration in terms of virtual source and partial derivative wavefields: Journal of Seismic Exploration, 12, 17-37.

Shin, C., and H. Sohn, 1998, A frequency-space 2D scalar wave extrapolator using extended 25-point finite difference operator: Geophysics, 63 289-296.

Shin, C., K. G. Yoon, K. J. Marfurt, K. P. Park, D. W. Yang, H. Y. Lim, S. H. Chung, and S. R. Shin, 2001b, Efficient calculation of a partial derivative wavefield using reciprocity for seismic imaging and inversion: Geophysics, 66, 1856-1863.

Song, Z.-M., P. R. Williamson, and R. G. Pratt, 1995, Frequency-domain acoustic-wave modeling and inversion of crosshole data: Part II: Inversion method, synthetic experiments and real-data results: Geophysics,
60, 796-809.

Sun, R., and G. A. McMechan, 1992, Full-wavefield inversion of wideaperture SH and Love wave data: Geophysical Journal International, 111, $1-10$.

Tarantola, A., 1984, Inversion of seismic reflection data in the acoustic approximation: Geophysics, 49, 1259-1266.

1986, A strategy for nonlinear elastic inversion of seismic reflection data: Geophysics, 51, 1893-1903.

, 1987, Inverse problem theory: Methods for data fitting and parameter estimation: Elsevier Science.

Tarantola, A., G. Jobert, D. Trezeguet, and E. Denelle, 1988, The nonlinear inversion of seismic waveforms can be performed either by time extrapolation or by depth extrapolation: Geophysical Prospecting, 36, 383-416.

Versteeg, R., 1994, The Marmousi experience: Velocity model determination on a synthetic complex data set: The Leading Edge, 13, 927-936.

Xu, T., G. A. McMechan, and R. Sun, 1995, 3D prestack full-wavefield inversion: Geophysics, 60, 1805-1818.

Zhou, C., W. Cai, Y. Luo, G. T. Schuster, and S. Hassanzadeh, 1995, Acoustic wave equation traveltime and waveform inversion of cross hole seismic data: Geophysics, 60, 765-773. 